\title{
Insights into the Changing Perspectives of Multiple Sclerosis in India
}

\author{
Lekha Pandit \\ Department of Neurology, KS Hegde Medical Academy, Nitte University, Mangalore 575018, Karnataka, India \\ Correspondence should be addressed to Lekha Pandit, panditmng@gmail.com \\ Received 7 January 2011; Revised 24 March 2011; Accepted 10 April 2011 \\ Academic Editor: D. N. Bourdette
}

Copyright ( 2011 Lekha Pandit. This is an open access article distributed under the Creative Commons Attribution License, which permits unrestricted use, distribution, and reproduction in any medium, provided the original work is properly cited.

Multiple Sclerosis (MS) is being diagnosed in increasing numbers in metropolitan cities of India for which the availability of specialist neurologists and magnetic resonance imaging (MRI) facilities are primarily responsible. Epidemiological data are unavailable. Existing data have been obtained from small often retrospective studies from different parts of the country. These earlier studies suggested that optic nerve and spinal cord involvement are considerably high, and that perhaps optic spinal MS was the most prevalent form in India. On this basis it was also speculated that neuromyelitis optica (NMO) may be overrepresented in Indians. However in recent times, prospective studies backed by MRI data have shown no distinct differences between MS seen in the west and India. Sero positivity for NMO IgG is low though NMO phenotype disorders constitute nearly $20 \%$ of demyelinating disorders in India. Genetic susceptibility for MS among Indians may be similar to that for white populations. In the major histocompatibility complex (MHC), HLA DR1*1501 has been strongly associated with MS in Indians. A recent study that evaluated the established non-MHC multiple sclerosis loci in a small data set of Indian patients suggested a strong similarity with white populations. This review highlights some of the background information available on MS from India and so also some recent studies that unveiled the disease characteristics in Indian patients.

\section{Introduction}

In the mid seventies in Kurtzke's MS world map [1] India was included in the list of countries belonging to the low prevalence zone $(<5 / 100,000)$. Even at that time, it was believed that in developing countries there was underestimation of disease prevalence for MS. Poser [2] mentioned paucity of neurologists, paucity of postmortem examinations, and unavailability of confirmatory test procedures such as neuroimaging, evoked potential studies, and reliable cerebrospinal fluid examination as some of the several problems in analyzing data from tropical countries. Record keeping and long-term followup of patients have seen serious limitations in all but the teaching hospitals and continue to hinder data collection and analysis of diseases in India. A decade later, there has been indirect evidence from hospital statistics in India suggesting that the number of cases diagnosed per year may have nearly doubled [3, 4]. This may be partly due to early detection especially since in recent times, MRI has become mandatory in all teaching hospitals in the country. Additionally there has been an increase in neurologists and also greater awareness which has resulted in white matter diseases including MS being increasingly diagnosed. In India public/teaching hospitals generally see patients from a lower socioeconomic background who visit hospitals with severe disease, males being given priority over females for seeking medical attention. These factors invariably hamper quality of data acquired from such settings.

\section{Prevalence of MS in India}

Neuroepidemiological studies have been done in the southern parts of the country and have failed to capture MS cases in the community [5]. A crude prevalence of approximately $1.33 / 100,000$ was reported by Singhal [6] in the mid eighties from the west coast of India. Hospital-based studies in India have shown that over the last decade, the total proportion of MS-related neurology department admissions increased from $1.58 \%$ to $2.54 \%$ [4]. This data is supported by the 
Multiple Sclerosis International Federation World MS Atlas, which projects a prevalence of $3 / 100,000$, which is nearly triple the estimate of previous reports. Geographically north west India (above $15^{\circ} \mathrm{N}$ latitude) saw $4.15 \mathrm{new}$ cases of MS per year as compared to 3.2 cases per year from south India (below $15^{\circ} \mathrm{N}$ latitude) [7]. The one exception is the Parsi population of India in whom Wadia and Bhatia [8] observed a prevalence of 26/100,000. The Parsis (Zorastrians) originated from the Pars province of Iran. Recent epidemiological studies have shown a high prevalence of MS in Iran especially in Ishfahan province [9] that adjoins Pars.

\section{Multiple Sclerosis in India; Phenotypic Differences from Western Population}

Early literature on MS from different parts of India suggested that there was high prevalence of optic and spinal cord involvement. In 1985 Jain and Maheshwari [7] published data on 354 cases of MS from 9 different centers in India. Optic neuritis (OPN) as the initial presentation was seen in $22.2-58 \%$ of cases seen at 5 of these centers. Recent studies have shown a frequency of $23.6 \%$ from the north west [10], $44 \%$ from the south of the country [11], and $53.3 \%$ [12] from the east of the country which is significantly high as compared to western data. In patients of North American and European origin optic neuritis was noted to be the initial presentation in only $17.2 \%$ of patients [13]. Acute onset of motor weakness was the next common initial presentation seen in $27-31 \%$ of Indian patients [10, 12]. A comparison in symptom at onset in patients admitted to a national hospital in south India before and after the mid eighties showed a dramatic reduction in spinal cord dysfunction by as much as $50 \%$ [11]. The authors attribute it to better awareness, early investigations including MRI and earlier diagnosis when patients present with milder symptoms.

\section{Neuromyelitis Optica (NMO) among Indian Demyelinating Disorders}

In earlier literature on MS in India $[6,7]$ the diagnosis of NMO was based on the descriptions by Shibasaki et al. [14]. It was limited to patients who showed "acute bilateral visual impairment and transverse spinal cord lesions, either concomitantly or within a short interval with or without subsequent improvement." The last decade has seen a major change in perception of NMO with a number of revisions in the diagnostic criteria [15-17]. Seen predominantly in middle aged women, this disorder is characterized by recurrent and severe involvement of optic nerve and spinal cord, with longitudinal spinal cord lesions and atypical brain lesions. Therein lies the dilemma in understanding how common it is among Indian CNS demyelinating disorders, wherein optic spinal presentations are common. Winger chuck et al. [17] have compiled available literature on NMO in India which included 59 cases reported between 1950 and 2006. They constituted roughly $9-24 \%$ of all demyelinating disorders seen in each series. There is not enough data available nor long-term followup on these patients to suggest there was severe visual loss or disproportionate spinal cord dysfunction in the subset with optic spinal presentation. In studies backed by MRI data [17, 18], MRI morphological data including length of spinal cord segments involved is not available. The high frequency of optic and spinal involvement cannot be therefore loosely translated into a possible high prevalence of NMO. Jain and Maheshwari [7] compilation of 354 cases of MS has been widely quoted in western literature as evidence for high prevalence of NMO in India. In their series $22-58 \%$ of patients reported had attacks confined to the spinal cord and optic nerve. The authors have noted that $33 / 354$ cases $(10 \%)$ had neuromyelitis optica as defined by Shibasaki et al. [14]. It is arguable that this definition was restrictive and there could have been higher numbers of NMO if considered in the light of current diagnostic criteria. This is not likely the case as shown by contemporary studies involving Indian patients, which correlated clinical presentation in MS alongside detailed MRI data. Mani et al. [19] compared 81 Indian with American MS patients matched for age, gender and duration of MS. There were no significant clinical or radiological differences between the two groups. NMO constituted $6 \%$ of Indian cases though $44 \%$ of patients in their Indian series had optic spinal presentation. In a prospective and longitudinal study of CNS demyelinating disorders which included 51 patients, Pandit et al. [20] found $47 \%$ of their MS cases have clinical attacks confined to the optic nerve and spinal cord. Yet NMO was diagnosed (Winger chuck et al. criteria [16] 1999) in only $5 \%$.

Neuromyelitis optica Immunoglobulin G (NMO IgG) seropostivity in Indian patients has been reported [21]. Pandit et al. analyzed NMO-IgG status in 78 consecutive cases obtained from their demyelination registry. In this study $62 / 78$ (80\%) patients belonged to the NMO spectrum. Longitudinally extensive transverse myelitis was seen in $39 / 78(50 \%)$ and included all cases of NMO, ATM, and recurrent ATM. Neuromyelitis optica-IgG was positive in $3 / 78$ patients $(3.8 \%)$, one each of NMO, optic spinal MS and recurrent acute transverse myelitis. Seropositive patients were all women and had late onset disease. Two of the three patients had optic neuritis and severe visual impairment and became wheel chair bound within 5 years of disease onset. Seronegative patients with long cord involvement were 36/78 (46\%). They had a male/female ratio of $2: 1$. Assistance for mobility was required by $12 / 36$ (33\%). The remaining $24 / 78(66.7 \%)$ patients who were seronegative and had long spinal cord lesions recovered well between attacks and remained ambulant $4-7$ years after disease onset [22]. A similar benign outcome has been described for NMO phenotype disorders in one other Indian study. Pradhan and Mishra [23] described 6 patients ( 3 male and 3 female) with recurrent optic neuritis and myelitis with documented long cord lesions on MRI of the spinal cord and normal MRI brain. These patients followed up for 2-10 years had good recovery in visual functions following OPN with only one patient requiring assistance to walk. Current data therefore appears to indicate that although optic and spinal cord presentations of CNS demyelinating diseases may be 
common in India, NMO meeting current diagnostic criteria may be no more common in India than in the west.

\section{Environmental Factors Implicated in MS in India}

Studies have shown that migration from low MS areas to high MS areas before the age of 15 increased the risk of MS in nonwhite populations, including Indians $[24,25]$. It is now clear that the rate of multiple sclerosis in second generation (UK born) Asians and first generation Asians coming to the UK as children, is significantly higher than in first generation (migrant) adult individuals. Ethnicity may confer some degree of protection against the increased risk. Thus in North America and the UK, MS increases among immigrants from India, while it remains low in immigrants from East Asia [26]. Geographical data indicates that MS is more common as distance from equator increases. This geographic variation may at least in part be attributable to environmental factors and be considered a reason for lower prevalence among Indians.

According to the "hygiene hypothesis" proposed in the 1960 's [27] children in areas where MS is rare are more likely to show evidence of childhood viral infections. Higher prevalence of MS is seen where sanitation and socioeconomic status are good. In this context, Singhal and Wadia [28] in their study of 30 patients from the Mumbai region observed that MS patients were mostly from higher socioeconomic status. Parsis of India in whom MS is highly prevalent are also an affluent community. A strong argument against the hygiene concept is that the affluent would have better opportunities to seek specialist care and have a diagnosis for their disease.

There are no large-scale studies which addressed specific environmental factors related to MS. Khadilkar et al. [29] have compared 63 patients with clinically definite MS with age, and sex-matched controls and evaluated the role of childhood viral infections, diet, evidence of concomitant autoimmune disorders, and smoking. Their study showed a significant association in history of childhood mumps $(P<$ .01 , odds ratio $(\mathrm{OR})=3$, confidence interval $(\mathrm{CI})=1.3-$ $7.2)$, and positive history of autoimmune diseases $(P<.05$, $\mathrm{OR}=2.8, \mathrm{CI}=1.2-6.8)$ among Indians with MS. There was a trend for association of MS among smokers in that 7 out of 10 smokers in Khadilakar's data set had MS.

\section{Genetic Susceptibility for MS in Indians}

The association between susceptibility to multiple sclerosis and the human leukocyte antigen (HLA) genes has been extensively studied in European and migrant European populations but the role of these genes in Asian (especially from the Indian subcontinent) multiple sclerosis has received almost no attention. The HLA allele frequencies found in this population, and the patterns of correlation (linkage disequilibrium) between these alleles is substantially different from that seen in other more extensively studied ethnic groups emphasising the potentially informative nature of a study of these genes in Asians. A few small serological studies considering the class I genes were performed in the 1980's and found association with HLA-B12 [30, 31] rather than B7. Subsequently analysis of the class II HLA genes, DRB1, DQA1, and DQB1 was attempted in a limited number of patients and revealed the expected association with the European susceptibility haplotype DRB1*1501$\mathrm{DQB} 1 * 0602[32,33]$. In considering the merits of this population in connection with the genetic analysis of multiple sclerosis it is relevant to note that although DR2 is actually more common in Asians than in Europeans, the DRB1*1501 allele (the main subtype of DR2 in Europeans) is notably less common (allele frequency $8 \%$ in Asians as compared to $15 \%$ in Europeans) (http://www.allelefrequencies.net/). In Asians the other subtypes of DR2 (the DRB1*1502 and $\mathrm{DRB} 1 * 16$ alleles) are more common and the extent of linkage disequilibrium (LD) between DRB1*1501 and DQB1 $* 0602$ is significantly less intense [34]. As described above populations where the established risk allele (haplotype) is uncommon have been especially informative regarding the effects attributable to other alleles and loci [35]. Studying populations with alternate patterns of LD have been helpful in disentangling the effects of linked loci [36]. These factors suggest that a genetic analysis of the $\mathrm{MHC}$ region in Asian patients is likely to be highly informative. Recently efforts have been focused on evaluating the role of established nonMHC disease susceptibility loci in Indian population [37]. Fifteen MS loci outside of the major histocompatibility (MHC) region that were previously identified and validated with MS susceptibility through genomewide association and replication studies in white populations were studied in 197 patients and unrelated controls. Despite the low power of the study, nominal associations were seen especially with interleukin-7 receptor alpha (IL7RA) and C type lectin domain family 16-(CLEC16 A-) associated single nucleotide polymorphisms (SNP's) and the risk allele frequencies in Indian Asian and white MS patients were comparable for most SNP's types. These preliminary observations suggest a commonality in disease susceptibility genes for both Indian and white populations.

\section{Conclusions}

Despite a modest estimated prevalence of MS in India of $3 / 100,000$, in this a nation of 1.2 billion people, there are probably more than 30,000 people with MS. With the currently available data, it appears that MS in India and in the west have similar presentations and risk factors. Although the role of selected environmental factors that confer susceptibility await evaluation, evidence suggests that genetic susceptibility factors may be similar in Indian and western populations, although this requires validation through larger studies. Collaborative efforts among specialist neurologists could help establish MS disease registries in various parts of the country to determine the longitudinal and natural history of this disease in India. Larger numbers would also increase the power of studies of genetic and environmental influences on MS in India. 


\section{References}

[1] J. F. Kurtzke, "Epidemiology of multiple sclerosis," in Handbook of Clinical Neurology. Vol. 3 Demyelinating Diseases, J. C. Koetssier, Ed., pp. 259-287, Elsevier Science, Amsterdam, The Netherlands, 1985.

[2] C. Poser, "Multiple sclerosis," in Tropical Neurology, R. Shakir, P. Newman, and C. Poser, Eds., pp. 437-455, WB Saunders, Philadelphia, Pa, USA, 1996.

[3] J. S. Chopra, K. Radhakrishnan, B. B. Sawhney, S. R. Pal, and A. K. Banerjee, "Multiple sclerosis in North-West India," Acta Neurologica Scandinavica, vol. 62, no. 5, pp. 312-321, 1980.

[4] P. Syal, S. Prabhakar, A. Thussu, S. Sehgal, and N. Khandelwal, "Clinical profile of multiple sclerosis in North-West India," Neurology India, vol. 47, no. 1, pp. 12-17, 1999.

[5] M. Gourie Devi, V. N. Rao, and R. Prakash, "Neuroepidemiological study in semi-urban and rural areas in South India: pattern of neurological disorders including motor neurone disease," in Motor Neurone Disease Global Clinical Patterns and International Research, M. Gourie Devi, Ed., pp. 11-21, Oxford and IBH, New Delhi, India, 1987.

[6] B. S. Singhal, "Multiple sclerosis-Indian experience," Annals of the Academy of Medicine Singapore, vol. 14, no. 1, pp. 32-36, 1985.

[7] S. Jain and M. C. Maheshwari, "Multiple sclerosis: Indian experience in the last thirty years," Neuroepidemiology, vol. 4, no. 2, pp. 96-107, 1985.

[8] N. Wadia and K. Bhatia, "Multiple sclerosis is prevalent in the Zoroastrians (Parsis) of India," Annals of Neurology, vol. 28, no. 2, pp. 177-179, 1990.

[9] M. Etemadifar, M. Janghorbani, V. Shaygannejad, and F. Ashtari, "Prevalence of multiple sclerosis in Isfahan, Iran," Neuroepidemiology, vol. 27, no. 1, pp. 39-44, 2006.

[10] P. Syal, S. Prabhakar, A. Thussu, S. Sehgal, and N. Khandelwal, "Clinical profile of multiple sclerosis in North-West India," Neurology India, vol. 47, no. 1, pp. 12-17, 1999.

[11] G. R. K. Sarma and D. K. Nagaraj, "Multiple sclerosis in South India," Annals of Indian Academy of Neurology, vol. 8, pp. 7174, 2005.

[12] G. Gangopadhyay, S. K. Das, P. Sarda et al., "Clinical profile of multliple sclerosis in Bengal,” Neurology India, vol. 47, no. 1, pp. 18-21, 1999.

[13] B. G. Weinshenker, B. Bass, G. P. A. Rice et al., "The natural history of multiple sclerosis: a geographically based study," Brain, vol. 112, no. 1, pp. 133-146, 1989.

[14] H. Shibasaki, Y. Kuroda, and Y. Kuroiwa, "Clinical studies of multiple sclerosis in Japan: classical multiple sclerosis and Devic's disease," Journal of the Neurological Sciences, vol. 23, no. 2, pp. 215-222, 1974.

[15] V. A. Lennon, D. M. Wingerchuk, T. J. Kryzer et al., "A serum autoantibody marker of neuromyelitis optica: distinction from multiple sclerosis," The Lancet, vol. 364, no. 9451, pp. 21062112, 2004.

[16] D. M. Wingerchuk, W. F. Hogancamp, P. C. O'Brien, and B. G. Weinshenker, "The clinical course of neuromyelitis optica (Devic's syndrome)," Neurology, vol. 53, no. 5, pp. 1107-1114, 1999.

[17] D. M. Wingerchuk, V. A. Lennon, C. F. Lucchinetti, S. Pittock, and B. G. Weinshenker, "The spectrum of neuromyelitis optica," Lancet Neurology, vol. 6, no. 9, pp. 805-815, 2007.

[18] A. Jacob and M. Boggild, "Neuromyelitis optica," Annals of Indian Academy of Neurology, vol. 10, no. 4, pp. 231-239, 2007.
[19] J. Mani, N. Chaudhary, S. Ravat, and P. U. Shah, "Multiple sclerosis: experience in neuroimaging era from western India," Neurology India, vol. 47, no. 1, pp. 8-11, 1999.

[20] L. Pandit, R. Shetty, I. G. Bhat, Z. Misri, and S. Hegde, "Spectrum of Multiple Sclerosis and related demyelinating disorders in India in the background of revised diagnostic criteria," Annals of Indian Academy of Neurology, vol. 10, supplement 2, pp. 44-45, 2007.

[21] L. Pandit, "Neuromyelitis optica antibody (NMO-IgG) status in Indian patients with multiple sclerosis and allied demyelinating disorders," Neurology Asia, vol. 13, no. 2, pp. 175-178, 2008.

[22] L. Pandit, R. Shetty, I. G. Bhat, Z. Misri, and S. Hegde, "Seronegative neuromyelitis spectrum disorders in India," Multiple Sclerosis, vol. 5, pp. S151-S269, 2009.

[23] S. Pradhan and V. N. Mishra, "A central demyelinating disease with atypical features," Multiple Sclerosis, vol. 10, no. 3, pp. 308-315, 2004.

[24] M. Elian, S. Nightingale, and G. Dean, "Multiple sclerosis among United Kingdom-born children of immigrants from the Indian subcontinent, Africa and the West Indies," Journal of Neurology Neurosurgery and Psychiatry, vol. 53, no. 10, pp. 906-911, 1990.

[25] G. Dean and M. Elian, "Age at immigration to England of Asian and Caribbean immigrants and the risk of developing multiple sclerosis," Journal of Neurology Neurosurgery and Psychiatry, vol. 63, no. 5, pp. 565-568, 1997.

[26] G. C. Ebers, "Environmental factors and multiple sclerosis," The Lancet Neurology, vol. 7, no. 3, pp. 268-277, 2008.

[27] U. Leibowitz, A. Antonovsky, J. M. Medalie, H. A. Smith, L. Halpern, and M. Alter, "Epidemiological study of multiple sclerosis in Israel. II. Multiple sclerosis and level of sanitation," Journal of Neurology Neurosurgery and Psychiatry, vol. 29, no. 1, pp. 60-68, 1966.

[28] B. S. Singhal and N. H. Wadia, "Profile of multiple sclerosis in the Bombay region: on the basis of critical clinical appraisal," Journal of the Neurological Sciences, vol. 26, no. 2, pp. 259-270, 1975.

[29] S. Khadilkar, A. O. Sahni, and S. A. Agarwal, "A case control study of environmental risk factors in Indians with multiple sclerosis," Neurophasia, vol. 10, pp. 47-52, 2005.

[30] N. H. Wadia, V. S. Trikannad, and P. R. Krishnaswamy, "HLA antigens in multiple sclerosis amongst Indians," Journal of Neurology Neurosurgery and Psychiatry, vol. 44, no. 9, pp. 849$851,1981$.

[31] V. S. Trikannad, N. H. Wadia, and P. R. Krishnaswamy, "Multiple sclerosis and HLA-B12 in Parsi and non-Parsi Indians. A clarification," Tissue Antigens, vol. 19, no. 2, pp. 155-157, 1982.

[32] M. A. Kelly, K. H. Jacobs, M. A. Penny et al., "An investigation of HLA-encoded genetic susceptibility to multiple sclerosis in subjects of Asian Indian and Afro-Caribbean ethnic origin," Tissue Antigens, vol. 45, no. 3, pp. 197-202, 1995.

[33] S. Kankonkar, G. Jeyanti, B. S. Singhal, and U. Shankarkumar, "Evidence for novel DRB1*15 allele association among clinically definite multiple sclerosis patients from Mumbai, India," Human Immunology, vol. 64, no. 4, pp. 478-482, 2003.

[34] R. Rani, M. A. Fernandez-Vina, and P. Stastny, "Associations between HLA class 11 alleles in a north Indian population," Tissue Antigens, vol. 52, no. 1, pp. 37-43, 1998.

[35] M. G. Marrosu, R. Murru, M. R. Murru, G. Costa, P. Zavattari, M. Whalen et al., "Dissection of the HLA association with multiple sclerosis in the founder isolated population 
of Sardinia," Human Molecular Genetics, vol. 10, no. 25, pp. 2907-2916, 2001.

[36] J. R. Oksenberg, L. F. Barcellos, B. A. Cree, S. E. Baranzini, T. L. Bugawan, O. Khan et al., "Mapping multiple sclerosis susceptibility to the HLA-DR locus in African Americans," American Journal of Human Genetics, vol. 74, no. 1, pp. 160167, 2004.

[37] L. Pandit, M. Ban, B. Singhal, S. Nair, K. Radhakrishnan, R. Shetty et al., "Evaluation of the established non-MHC multiple sclerosis loci in an Indian population," Multiple Sclerosis, vol. 17, no. 2, pp. 139-143, 2011. 


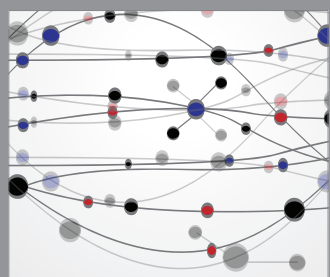

The Scientific World Journal
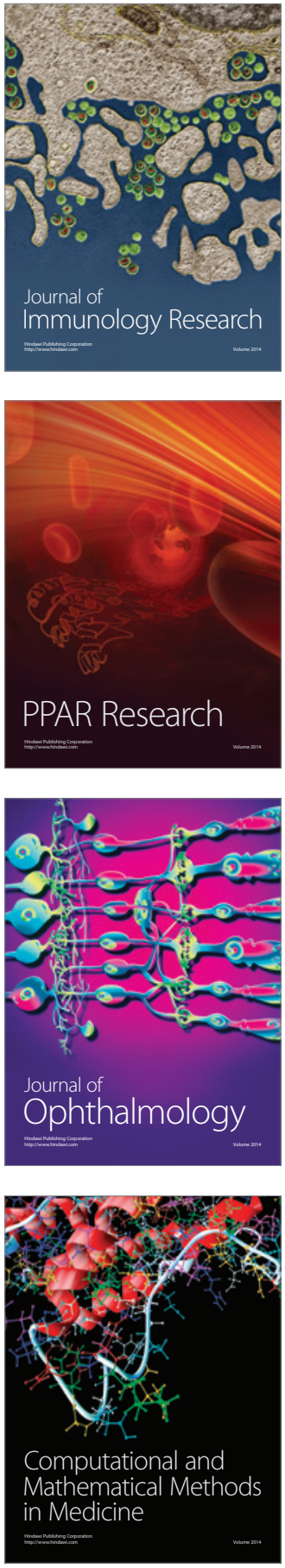

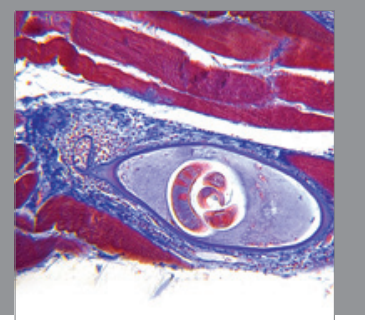

Gastroenterology

Research and Practice
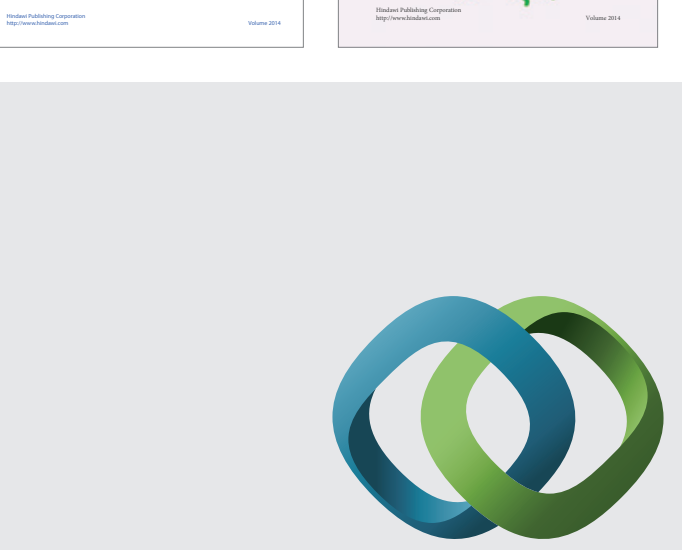

\section{Hindawi}

Submit your manuscripts at

http://www.hindawi.com
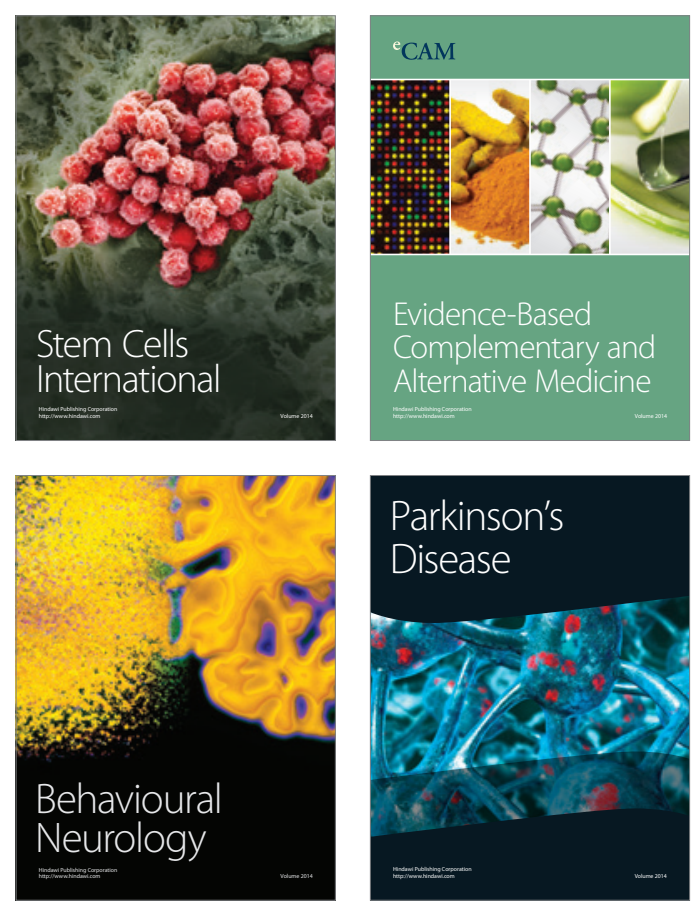

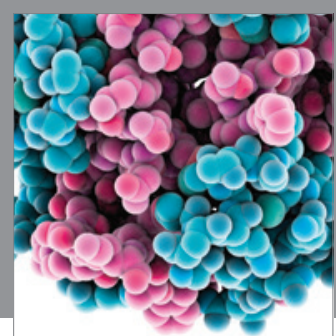

Journal of
Diabetes Research

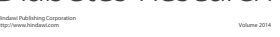

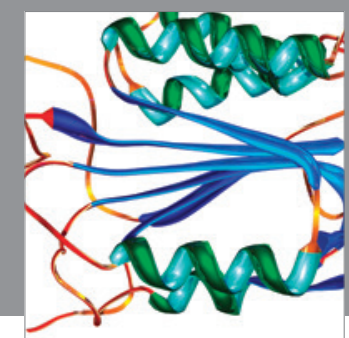

Disease Markers
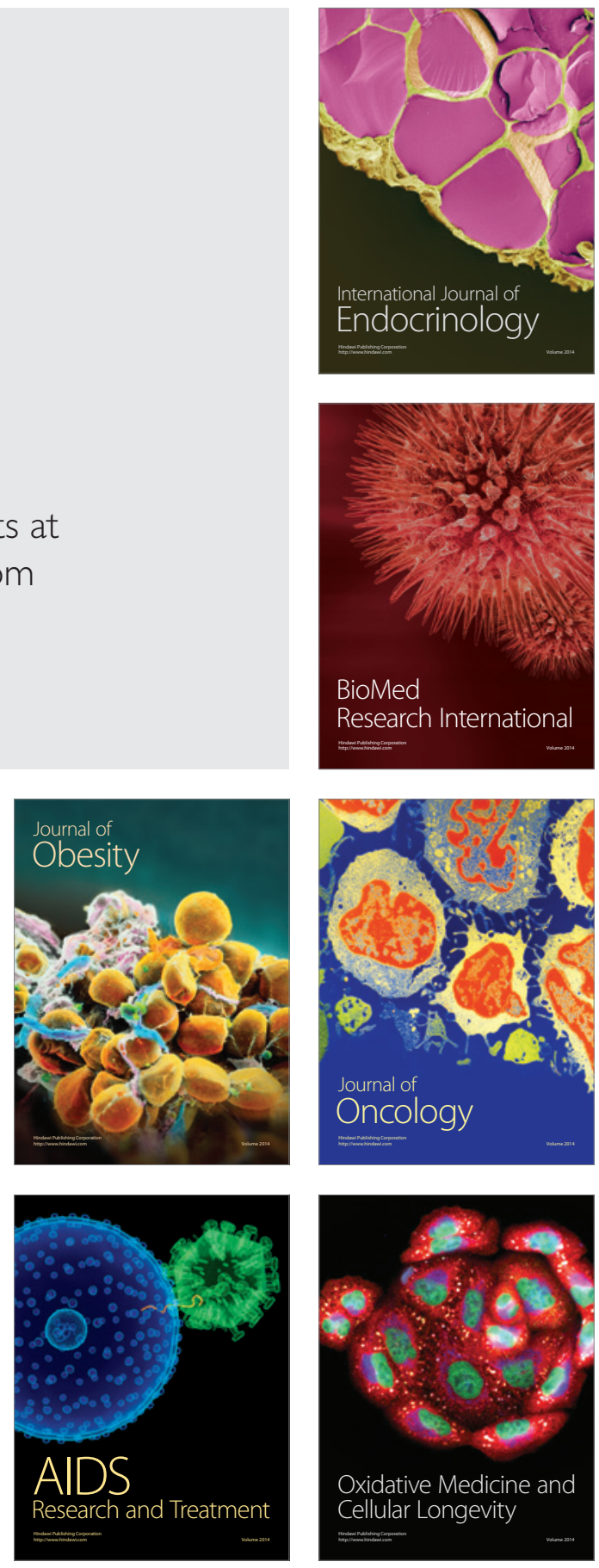